\title{
Real-time recurrent learning neural network for stream-flow forecasting
}

\author{
Fi-John Chang, ${ }^{*}$ Li-Chiu Chang and Hau-Lung Huang \\ Department of Bioenvironmental Systems Engineering, National Taiwan University, Taipei, Taiwan, ROC
}

\begin{abstract}
:
Various types of neural networks have been proposed in previous papers for applications in hydrological events. However, most of these applied neural networks are classified as static neural networks, which are based on batch processes that update action only after the whole training data set has been presented. The time variate characteristics in hydrological processes have not been modelled well. In this paper, we present an alternative approach using an artificial neural network, termed real-time recurrent learning (RTRL) for stream-flow forecasting. To define the properties of the RTRL algorithm, we first compare the predictive ability of RTRL with least-square estimated autoregressive integrated moving average models on several synthetic time-series. Our results demonstrate that the RTRL network has a learning capacity with high efficiency and is an adequate model for time-series prediction. We also investigated the RTRL network by using the rainfall-runoff data of the Da-Chia River in Taiwan. The results show that RTRL can be applied with high accuracy to the study of real-time stream-flow forecasting networks. Copyright (C) 2002 John Wiley \& Sons, Ltd.
\end{abstract}

KEY WORDS recurrent neural networks; stream-flow forecasting; rainfall-runoff modelling

\section{INTRODUCTION}

Building a real-time stream-flow forecasting model has always been one of the most challenging and important tasks for hydrologists in Taiwan, which has the attributes of subtropical climate and high mountains with steep slopes all over the island. In addition, typhoons hit Taiwan around four times a year, bringing heavy rainfalls, which can flood downstream cities within a few hours. Owing to these particular watershed-rainfall characteristics, accurate site-specific predictions of the real-time stream-flow remain a difficult task. Various types of deterministic and stochastic models, as described by Sherman (1932), the Hydrological Engineering Center (1990) and Salas et al. (1985) have been used to construct the rainfall-runoff processes in Taiwan. However, some unrealistic assumptions and the lack of verified data/parameters in these models have limited their application and practicality.

Capable of modelling non-linear and complex systems, artificial neural networks (ANNs) provide an alternative approach for accurate stream-flow forecasting. Generally speaking, neural networks are information processing systems devised via imitating brain activity. After McCulloch and Pitts (1943) established the first neural network, many ANNs, such as the back-propagation neural network (Rumelhart et al., 1986), the Hopfield neural network (Hopfield, 1984) and the fuzzy neural network (Nie and Linkens, 1994). were developed to solve different problems. More recently, they have been used to deal with stream-flow prediction (Hsu et al., 1995; Shamseldin, 1997; Chang and Hwang, 1999; Sajikumar and Thandareswara 1999; Chang et al., 2001; Chang and Chen, 2001). Although satisfactory results have been reported, the applied neural networks for hydrological processes, to the best of our knowledge, are all based on batch processes, by

* Correspondence to: Professor F.-J. Chang, Department of Bioenvironmental Systems Engineering and Hydrotech Research Institute, National Taiwan University, Taipei, Taiwan, ROC. E-mail: changfj@ccms.ntu.edu.tw 
which the update action takes place only after the whole training data set has been presented. These neural networks are classified as static neural networks, which can only simulate the short-term memory structures within processes. The extraordinary time variate characteristics of hydrological time series, especially in our case, could not be modelled well. Therefore, we present an alternative approach of the ANN for streamflow forecasting — the real-time recurrent learning (RTRL) algorithm (Williams and Zipser, 1989), which provides a representation of dynamic internal feedback loops to store information for later use and to enhance the efficiency of learning. The application of feedback enables recurrent networks to acquire state representation, which make them suitable devices for diverse applications, such as non-linear prediction, speech processing and plant control (Haykin, 1999).

We began with a series of simulation experiments to investigate the properties of the RTRL algorithm, and then implemented it to model a watershed rainfall-runoff process for real-time stream-flow forecasting.

\section{REAL-TIME RECURRENT LEARNING (RTRL) ALGORITHM}

Figure 1 shows the architecture of a multiplayer perceptron neural network, which includes a concatenated input-output layer, a processing layer and an output layer. There are $M$ external inputs and $K$ outputs. Let $\boldsymbol{x}(t)$ denote the $M \times 1$ input vector to the network at discrete time $t, z(t+1)$ denote the corresponding $K \times 1$ output vector and $\boldsymbol{y}(t+1)$ denote the corresponding $N \times 1$ vector one step later at time $t+1$ in the processing layer. The input $\boldsymbol{x}(t)$ and one-step delayed output vector in the processing layer $\boldsymbol{y}(t)$ are concatenated to form the $(M+N) \times 1$ vector $\boldsymbol{\mu}(t)$, in which the $i$ th element is denoted by $\boldsymbol{\mu}_{i}(t)$. Let $A$ denote the set of indices $i$ for which $\boldsymbol{x}_{i}(t)$ is an external input, and $B$ denote the set of indices $i$ for which $\boldsymbol{y}_{i}(t)$ is the output of a unit in the network. We thus have

$$
\mu_{i}(t)= \begin{cases}x_{i}(t) & \text { if } i \in A \\ y_{i}(t) & \text { if } i \in B\end{cases}
$$

The network is fully interconnected. There are $M \times N$ forward connections and $N \times N$ feedback connections. Let $\mathbf{W}$ denote $N \times(M+N)$, the recurrent weight matrix of the network. To allow each unit a bias weight, we simply include, among the $M$ input lines, one input with a value that is always $1 . \mathbf{W} \leftrightarrow w_{j i}$ and $\mathbf{V} \leftrightarrow v_{k j}$ are the matrix form.

The processing and output layers are also fully connected. Let $\mathbf{V}$ denote the $N \times K$ weight matrix.

The net activity of neuron $j$ at time $t$, for $j \in B$, is computed by

$$
\operatorname{net}_{j}(t)=\sum_{i \in A \cup B} w_{j i}(t-1) \mu_{i}(t-1)
$$

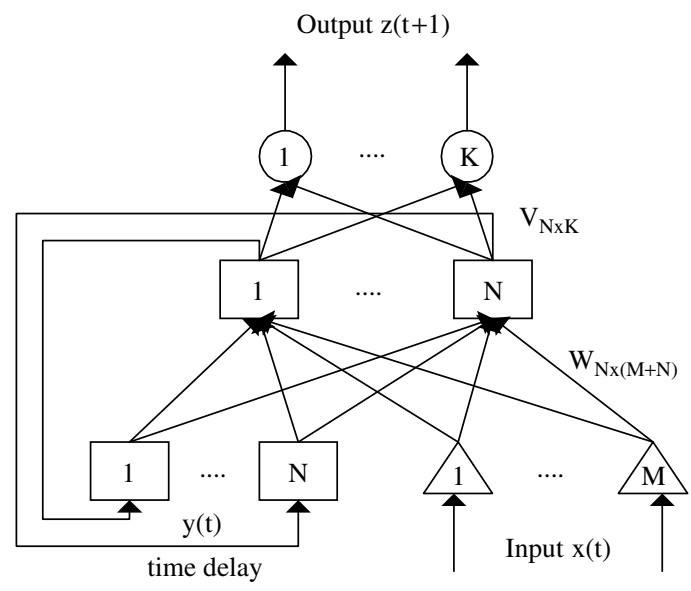

Figure 1. Architecture of RTRL network
Output Layer Processing Layer

Concatenated inputoutput Layer 
The output of neuron $j$ is given by passing net ${ }_{j}(t)$ through the non-linearity $f($.$) , yielding$

$$
\boldsymbol{y}_{j}(t)=f\left(\operatorname{net}_{j}(t)\right)
$$

The net output of neuron $k$ at time $t$ is computed by

$$
\begin{aligned}
\operatorname{net}_{k}(t) & =\sum v_{k j}(t) \boldsymbol{y}_{j}(t) \\
\boldsymbol{z}_{k}(t) & =f\left(\operatorname{net}_{k}(t)\right)
\end{aligned}
$$

Notice that the external input at time $t$ does not influence the output of any neuron until time $t+1$. The above system of equations constitutes the entire dynamics of the network.

Let $d_{k}(t)$ denote the target value of neuron $k$ at time $t$. Then we define a time-varying $K \times 1$ error vector $\boldsymbol{e}(t)$, whose $k$ th element is

$$
\boldsymbol{e}_{k}(t)=d_{k}(t)-z_{k}(t)
$$

Define the instantaneous overall network error at time $t$ as

$$
E(t)=\frac{1}{2} \sum_{k=1}^{K} \boldsymbol{e}_{k}^{2}(t)
$$

The cost function is obtained by summing $E(t)$ over all time $T$

$$
E_{\text {total }}=\sum_{t=1}^{T} E(t)
$$

To minimize the cost function, the gradient descent method is applied to adjust the weights ( $\mathbf{V}$ and $\mathbf{W}$ ) along the negative of $\nabla E_{\text {total }}$. Because the total error is the sum of the errors at the individual time steps, one way to compute this gradient is by accumulating the value of $\nabla E$ for each time step along the trajectory. The weight change for any particular weight $v_{k j}$ can thus be written as

$$
\begin{aligned}
\Delta \boldsymbol{V}_{k j} & =\sum_{t=t_{0}+1}^{t_{1}} \Delta v_{k j}(t) \\
\Delta v_{k j}(t) & =-\eta_{1} \frac{\partial E(t)}{\partial v_{k j}(t)}
\end{aligned}
$$

where $\eta_{1}$ is the learning-rate parameter. Now

$$
\frac{\partial E(t)}{\partial v_{k j}(t)}=-\boldsymbol{e}_{k}(t) f^{\prime}\left(\operatorname{net}_{k}(t)\right) \boldsymbol{y}_{j}(t)
$$

The same method is also implemented for weight $w_{m n}$, where

$$
\Delta w_{m n}(t-1)=-\eta_{2} \frac{\partial E(t)}{\partial w_{m n}(t-1)}
$$


and $\eta_{2}$ is the learning-rate parameter. The partial derivative $\frac{\partial E(t)}{\partial w_{m n}(t-1)}$ can be obtained by the chain rule for differentiation as follows

$$
\begin{aligned}
\frac{\partial E(t)}{\partial w_{m n}(t-1)} & =\left[\sum_{k=1}^{K}-\boldsymbol{e}_{k}(t) f^{\prime}\left(\operatorname{net}_{k}(t)\right) v_{k j}(t)\right] \frac{\partial \boldsymbol{y}_{j}(t)}{\partial w_{m n}(t-1)} \\
\Rightarrow \frac{\partial \boldsymbol{y}_{j}(t)}{\partial w_{m n}(t-1)} & =f^{\prime}\left(\operatorname{net}_{j}(t)\right) \frac{\partial \operatorname{net}_{j}(t)}{\partial w_{m n}(t-1)} \\
\Rightarrow \frac{\partial \text { net }_{j}(t)}{\partial w_{m n}(t-1)} & =\sum_{i \in A \cup B} \frac{\partial\left(w_{j i}(t-1) \boldsymbol{\mu}_{i}(t-1)\right)}{\partial w_{m n}(t-1)} \\
\Rightarrow \frac{\partial \text { net }_{j}(t)}{\partial w_{m n}(t-1)} & =\sum_{i \in A \cup B}\left[w_{j i}(t-1) \frac{\partial \boldsymbol{\mu}_{i}(t-1)}{\partial w_{m n}(t-1)}+\frac{\partial w_{j i}(t-1)}{\partial w_{m n}(t-1)} \boldsymbol{\mu}_{i}(t-1)\right]
\end{aligned}
$$

The derivative $\frac{\partial w_{j i}(t-1)}{\partial w_{m n}(t-1)}$ is equal to 1 only when $j=m$ and $i=n$; otherwise, it is zero. We may therefore rewrite the above equations as

$$
\frac{\partial \text { net }_{j}(t)}{\partial w_{m n}(t-1)}=\sum_{i \in A \cup B} w_{j i}(t-1) \frac{\partial \mu_{i}(t-1)}{\partial w_{m n}(t-1)}+\delta_{m j} \mu_{n}(t-1)
$$

where $\delta_{m j}$ is the Kronecker delta with value 1 if and only if $j=m$; otherwise zero.

From the definition of $\boldsymbol{\mu}_{i}(t)$, we also note that

$$
\frac{\partial \boldsymbol{\mu}_{i}(t-1)}{\partial w_{m n}(t-1)}=\left\{\begin{array}{cl}
0 & \text { if } i \in A \\
\frac{\partial \boldsymbol{y}_{i}(t-1)}{\partial w_{m n}(t-1)} & \text { if } i \in B
\end{array}\right.
$$

We assume that the initial state of the network at time $t=0$ has no functional dependence on the synaptic weights, so that

$$
\begin{aligned}
\frac{\partial \boldsymbol{y}_{j}(0)}{\partial w_{m n}(0)} & =0 \\
\frac{\partial \boldsymbol{y}_{j}(t)}{\partial w_{m n}(t-1)} & =f^{\prime}\left(\text { net }_{j}(t)\right)\left[\sum_{i \in B} w_{j i}(t-1) \frac{\partial y_{i}(t-1)}{\partial w_{m n}(t-1)}+\delta_{m j} \boldsymbol{\mu}_{n}(t-1)\right]
\end{aligned}
$$

Let $\frac{\partial \boldsymbol{y}_{j}(t)}{\partial w_{m n}(t)} \approx \frac{\partial \boldsymbol{y}_{j}(t)}{\partial w_{m n}(t-1)}$ and define a dynamic system described by a triple indexed set of variables $\left\{\pi_{m n}^{j}\right\}$, where $\pi_{m n}^{j}(t)=\frac{\partial \boldsymbol{y}_{j}(t)}{\partial w_{m n}(t)}$ for all $j \in B, m \in B$ and $n \in A \cup B$.

For each time step $t$ and all appropriate $m, n$ and $j$, the dynamics of the system are governed by

$$
\pi_{m n}^{j}(t)=f^{\prime}\left(\text { net }_{j}\right)\left[\sum_{i \in B} w_{j i}(t-1) \pi_{m n}^{i}(t-1)+\delta_{m j} \boldsymbol{u}_{n}(t-1)\right]
$$

with initial condition $\pi_{m n}^{j}(0)=0$. Then the weight changes can be computed as

$$
\begin{aligned}
\Delta w_{m n}(t-1) & =\eta_{2}\left[\sum \boldsymbol{e}_{k}(t) f^{\prime}\left(\operatorname{net}_{k}(t)\right) v_{k j}(t)\right] \pi_{m n}^{j}(t) \\
\Delta v_{k j}(t) & =\eta_{1} \boldsymbol{e}_{k}(t) f^{\prime}\left(\operatorname{net}_{k}(t)\right) \boldsymbol{y}_{j}(t)
\end{aligned}
$$




\section{SIMULATING SYNTHETIC TIME SERIES}

Statistical approach to the hydrological time-series forecasting involves constructing stochastic models to predict the value based on previous observations. This often is accomplished by using linear stochastic differential equation models with random inputs. By far the most profound models are the autoregressive integrated moving average (ARIMA) models. Detailed discussions and applications of these models may be found in Box and Jenkins (1976) and Salas et al. (1985). To show the robustness of RTRL, we compare the predictive ability of the least-squares estimated ARIMA predictors and RTRL on five different time-series synthetic data. The five time-series models are shown as:

1. $\operatorname{AR}(1)(x(t)-0.4)=0.75(x(t-1)-0.4)+\boldsymbol{e}_{t}$, with $\boldsymbol{e}_{t}^{\text {i.i.d }} \approx N(0,1 \cdot 0)$;

2. $\operatorname{AR}(2)(x(t)-0 \cdot 4)=0 \cdot 6(x(t-1)-0.4)+0 \cdot 2(x(t-2)-0 \cdot 4)+\boldsymbol{e}_{t}$, with $\boldsymbol{e}_{t}^{\text {i.i.d }} \approx N(0,1 \cdot 0)$;

3. $\operatorname{IMA}(1,0) x(t)=x(t-1)+\boldsymbol{e}_{t}$, with $\boldsymbol{e}_{t}^{\text {i.i.d }} \approx N(0,1 \cdot 0)$;

4. $\operatorname{IMA}(1,1)(1-B) x(t)=(1-0.6 B) \boldsymbol{e}_{t}$, with $\boldsymbol{e}_{t}^{\text {i.i.d }} N \approx(0,1 \cdot 0)$;

5. ARIMA $(1,1,1)(1-B) x(t)=0 \cdot 4(1-B) x(t-1)+\boldsymbol{e}_{t}-0 \cdot 3 \boldsymbol{e}_{t-1}$, with $\boldsymbol{e}_{t}^{\mathrm{i} i \mathrm{id}} \approx N(0,1 \cdot 0)$

The $\boldsymbol{e}_{t}$ values are chosen from an independent and identically normal distribution (i.i.d) with mean zero and variance one. Each series is generated by MATLAB the Maths Works, Inc. After the first 50 generated data are skipped, the initial 100 data are used to construct the RTRL network and to estimate the optimal parameters of the ARIMA models. For simplicity, without model identification, we use the given types of the ARIMA models, and only their parameters are estimated. The least-squares estimator in MATLAB is used to estimate the ARIMA models' parameters. The architecture of the RTRL network for one-step ahead prediction is illustrated in Figure 1. There is only one single input, the current value $\boldsymbol{x}(t)$, and the processing layer has been investigated through the training data set and identified to have five to seven nodes for different times series. Both learning rates $\left(\eta_{1}\right.$ and $\left.\eta_{2}\right)$ are 100.0. Because we only perform one-step ahead prediction, $\hat{\boldsymbol{x}}(t+1)$, the output node is, then, set as 1 .

The one-step ahead prediction is performed based on (i) the constructed RTRL networks and (ii) the fitted ARIMA models for each case, respectively. As the prediction focuses on optimum prediction by the minimum mean squared error, the criteria of mean square error (MSE) and mean absolute error (MAE) are used for the purpose of comparison.

To learn the models' performance at different stages, the models' predictions in (i) training sets (100 data) and (ii) testing sets (200 data) at three different periods are presented, respectively. The results are summarized in Tables I-V. To illustrate the models' performances in general, the series of ARIMA $(1,1,1)$ and its corresponding prediction errors by (i) the fitted ARIMA model and (ii) the constructed RTRL networks are shown in Figure 2. Apparently, the results show that the RTRL networks have very similar performances as those of the fitted ARIMA models and the networks could persist with the main statistical properties, in terms of reasonable MAE and MSE values, of the generated ARIMA series. The results of RTRL in the

Table I. The performance of fitted AR(1) and RTRL at different stages

\begin{tabular}{lcccccc}
\hline \multirow{2}{*}{ Set } & Time-step & \multicolumn{2}{c}{ AR(1) } & & \multicolumn{2}{c}{ RTRL } \\
\cline { 3 - 4 } \cline { 6 - 6 } & & MAE & MSE & & MAE & MSE \\
\hline Training & $1-30$ & 1.016 & 1.569 & & 1.640 & 4.906 \\
\multirow{3}{*}{ Testing } & $1-100$ & 0.836 & 1.107 & & 1.053 & 2.059 \\
& $101-130$ & 0.676 & 0.829 & & 0.821 & 1.152 \\
& $151-180$ & 0.741 & 0.848 & & 0.852 & 1.197 \\
& $271-300$ & 0.614 & 0.657 & & 0.667 & 0.789 \\
& $101-300$ & 0.722 & 0.876 & & 0.809 & 1.114 \\
\hline
\end{tabular}


Table II. The performance of fitted AR(2) and RTRL at different stages

\begin{tabular}{lcccccc}
\hline \multirow{2}{*}{ Set } & \multirow{2}{*}{ Time-step } & \multicolumn{2}{c}{ AR(2) } & & \multicolumn{2}{c}{ RTRL } \\
\cline { 3 - 4 } \cline { 6 - 6 } & & MAE & MSE & & MAE & MSE \\
\hline Training & $1-30$ & 0.879 & 1.155 & & 1.115 & 2.994 \\
\multirow{2}{*}{ Testing } & $1-100$ & 0.853 & 1.087 & & 1.015 & 1.626 \\
& $101-130$ & 0.698 & 0.733 & & 0.736 & 0.777 \\
& $151-180$ & 0.709 & 0.807 & & 0.787 & 0.955 \\
& $271-300$ & 0.620 & 0.700 & & 0.782 & 0.809 \\
& $101-300$ & 0.745 & 0.880 & & 0.846 & 1.114 \\
\hline
\end{tabular}

Table III. The performance of fitted IMA $(1,0)$ and RTRL at different stages

\begin{tabular}{|c|c|c|c|c|c|}
\hline \multirow[t]{2}{*}{ Set } & \multirow[t]{2}{*}{ Time-step } & \multicolumn{2}{|c|}{$\operatorname{IMA}(1,0)$} & \multicolumn{2}{|c|}{ RTRL } \\
\hline & & $\overline{\mathrm{MAE}}$ & $\overline{\mathrm{MSE}}$ & $\overline{\mathrm{MAE}}$ & MSE \\
\hline \multirow[t]{2}{*}{ Training } & $1-30$ & 0.721 & 0.737 & $1 \cdot 112$ & $3 \cdot 379$ \\
\hline & $1-100$ & 0.654 & 0.672 & 0.998 & $2 \cdot 770$ \\
\hline \multirow[t]{4}{*}{ Testing } & $101-130$ & 0.891 & $1 \cdot 1819$ & 0.976 & 1.594 \\
\hline & $151-180$ & 1.023 & 1.6276 & 1.033 & 1.588 \\
\hline & $271-300$ & 0.825 & $1 \cdot 0487$ & $0 \cdot 809$ & 1.011 \\
\hline & $101-300$ & 0.891 & $1 \cdot 2053$ & 0.923 & 1.271 \\
\hline
\end{tabular}

Table IV. The performance of fitted IMA $(1,1)$ and RTRL at different stages

\begin{tabular}{|c|c|c|c|c|c|}
\hline \multirow[t]{2}{*}{ Set } & \multirow[t]{2}{*}{ Time-step } & \multicolumn{2}{|c|}{$\operatorname{IMA}(1,1)$} & \multicolumn{2}{|c|}{ RTRL } \\
\hline & & $\overline{\mathrm{MAE}}$ & MSE & $\overline{\mathrm{MAE}}$ & MSE \\
\hline \multirow[t]{2}{*}{ Training } & $1-30$ & 0.878 & 1.097 & 1.627 & 11.735 \\
\hline & $1-100$ & 0.828 & 1.060 & 0.988 & 1.596 \\
\hline \multirow[t]{4}{*}{ Testing } & $101-130$ & 0.964 & 1.452 & 0.929 & 1.455 \\
\hline & $151-180$ & 0.951 & 1.278 & 0.931 & $1 \cdot 280$ \\
\hline & $271-300$ & 0.777 & 0.837 & 0.788 & $0 \cdot 830$ \\
\hline & $101-300$ & 0.821 & 1.096 & 0.853 & $1 \cdot 170$ \\
\hline
\end{tabular}

Table V. The performance of fitted ARIMA $(1,1,1)$ and RTRL at different stages

\begin{tabular}{lccccrr}
\hline \multirow{2}{*}{ Set } & Time-step & \multicolumn{2}{c}{ ARIMA $(1,1,1)$} & & \multicolumn{2}{c}{ RTRL } \\
\cline { 3 - 4 } \cline { 6 - 7 } & & MAE & MSE & & MAE & MSE \\
\hline Training & $1-30$ & 0.604 & 0.596 & & 1.686 & 12.828 \\
& $1-100$ & 0.790 & 0.966 & & 1.243 & 3.257 \\
Testing & $101-130$ & 0.772 & 0.880 & & 0.952 & 1.545 \\
& $151-180$ & 0.764 & 0.898 & & 0.742 & 0.871 \\
& $271-300$ & 0.797 & 0.909 & & 0.774 & 0.916 \\
& $101-300$ & 0.781 & 0.954 & & 0.808 & 1.059 \\
\hline
\end{tabular}



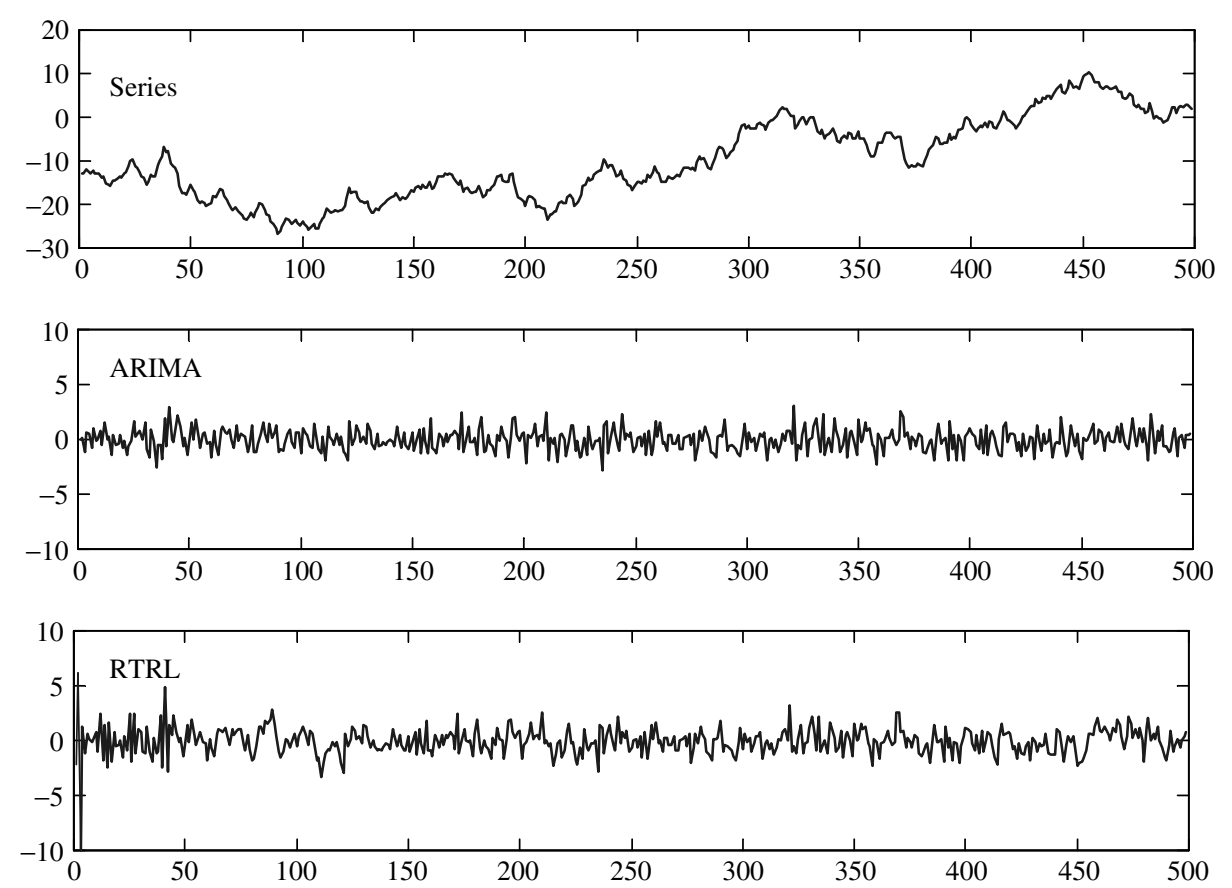

Figure 2. The series of ARIMA(1,1,1) and its corresponding errors using a fitted ARIMA model and a constructed RTRL network

training set indicate that after about 30 steps on-line input training, RTRL can grasp the major trends in all cases. All of them indicate that RTRL has an efficient ability to learn and a high accuracy.

\section{APPLICATION}

The above method is applied to the upstream of the Da-Chia River for predicting real-time stream flow. The Da-Chia River is located in central Taiwan and has a total catchment size of $1236 \mathrm{~km}^{2}$. Being the steepest channel in Taiwan, the Da-Chia River has a length of $140 \mathrm{~km}$ and an average channel slope of 1/39. A series of hydraulic structures were constructed for power generation. Locations of the basin studied $\left(\operatorname{area}=514 \mathrm{~km}^{2}\right.$ ) and gauge stations used are shown in Figure 3. A station for the stream-flow data is denoted by a triangle and the precipitation stations are shown as circles. Son-Mou gauge station was established to measure the inflow to the De-Chi Reservoir, the upmost and pivotal reservoir in the Da-Chia River. Accurate stream-flow forecasting is extremely important for the operation of the De-Chi Reservoir. The stream flow $\left(\mathrm{m}^{3} / \mathrm{s}\right)$ and precipitation $(\mathrm{mm} / \mathrm{h})$ data used here are gathered by the Taiwan Power Company.

There are four rainfall gauges above the Son-Mou stream-flow gauge. To construct a one-step ahead streamflow forecasting, we investigate the RTRL and ARMAX (Autoregressive moving average with exogenous inputs) models' performance based on current rainfall at four gauges and stream-flow data. The performances of these two methods are presented based on the criteria of annual peak-flow estimation, mean absolute error (MAE) and relative mean absolute error (RMAE) as shown below

$$
\mathrm{MAE}=\frac{\sum_{i=1}^{n}\left(\hat{Q}_{i}-Q_{i}\right)}{n}
$$




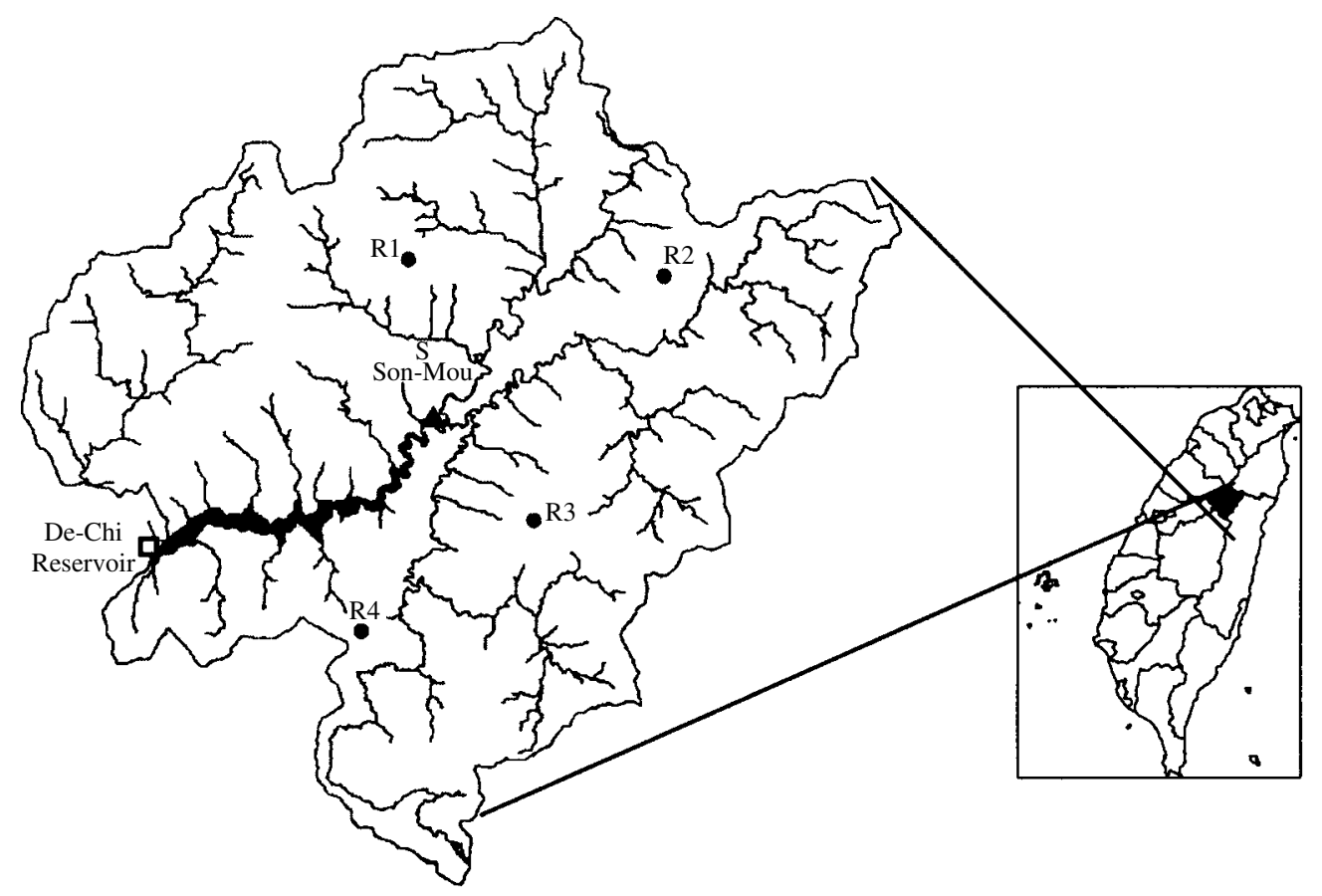

Figure 3. Location of gauge stations in the upstream part of the Da-Chia River

Table VI. Table of model results for different years: $Q_{\mathrm{o}}$ is annual mean of observed stream-flow (cms), $Q_{\mathrm{p}}$ is observed annual peak flow (cms) and $\hat{Q}_{\mathrm{p}}$ is estimated annual peak flow (cms)

\begin{tabular}{|c|c|c|c|c|c|c|c|c|}
\hline \multirow[t]{2}{*}{ Year } & \multirow[t]{2}{*}{$Q_{\mathrm{o}}$} & \multirow[t]{2}{*}{$Q_{\mathrm{p}}$} & \multicolumn{3}{|c|}{$\operatorname{ARMAX}(1,1,3)$} & \multicolumn{3}{|c|}{ RTRL } \\
\hline & & & $\overline{\mathrm{MAE}}$ & RMAE & $\hat{Q}_{\mathrm{p}}$ & $\overline{\mathrm{MAE}}$ & RMAE & $\hat{Q}_{\mathrm{p}}$ \\
\hline 1992 & $45 \cdot 87$ & 699 & 0.792 & 0.0173 & 695 & $0 \cdot 648$ & 0.0141 & 703 \\
\hline 1993 & $15 \cdot 85$ & 214 & $0 \cdot 215$ & 0.0136 & 194 & $0 \cdot 190$ & 0.0120 & 199 \\
\hline 1994 & 34.66 & 1120 & 0.930 & 0.0268 & 1075 & 0.741 & 0.0214 & 1190 \\
\hline 1995 & $19 \cdot 01$ & 105 & 0.239 & $0 \cdot 0126$ & 102 & 0.223 & 0.0118 & 102 \\
\hline 1996 & 24.04 & 1090 & 0.476 & 0.0198 & 1020 & 0.415 & 0.0172 & 1062 \\
\hline
\end{tabular}

The hourly data of 1992 are used to construct the ARMAX and the RTRL network. The ARMAX $(1,1,3)$ model is shown by the following

$$
\begin{aligned}
S(t)= & a_{1} S(t-1)+\sum_{i=1}^{3} b_{1 i} R 1(t-i)+\sum_{i=1}^{3} b_{2 i} R 2(t-i)+\sum_{i=1}^{3} b_{3 i} R 3(t-i) \\
& +\sum_{i=1}^{3} b_{4 i} R 4(t-i)+\boldsymbol{e}(t)+c_{1} \boldsymbol{e}(t-1)
\end{aligned}
$$

where $S(t)$ is stream flow of the Son-Mou stream-flow gauge at time $t ; R 1(t), R 2(t), R 3(t)$ and $R 4(t)$ are precipitation of four rainfall gauges at time $t ; \boldsymbol{e}(t)$ is the model error at time $t$; and $a_{1}, b_{i j}, c_{1}$ are the model's parameters to be estimated. The model and its parameters can be obtained by using MATLAB. 


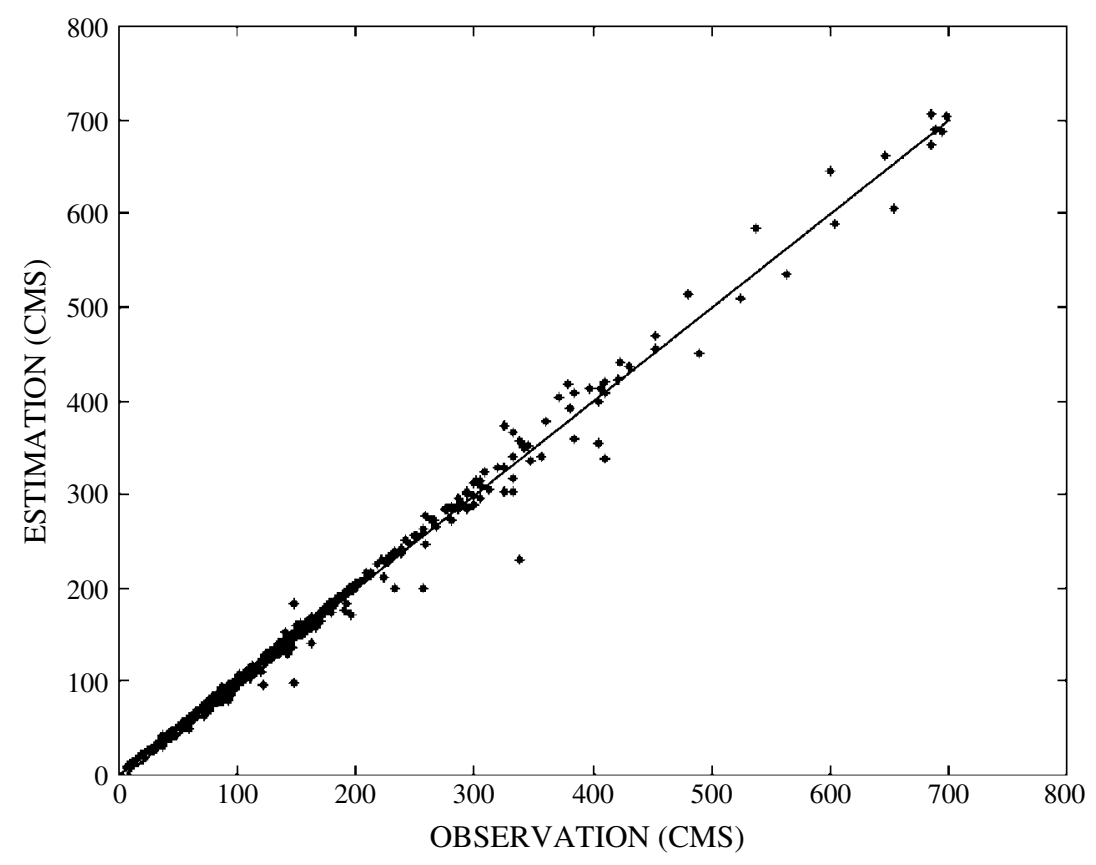

Figure 4. Comparison of observed and forecast stream flow at the Son-Mou gauge in 1992

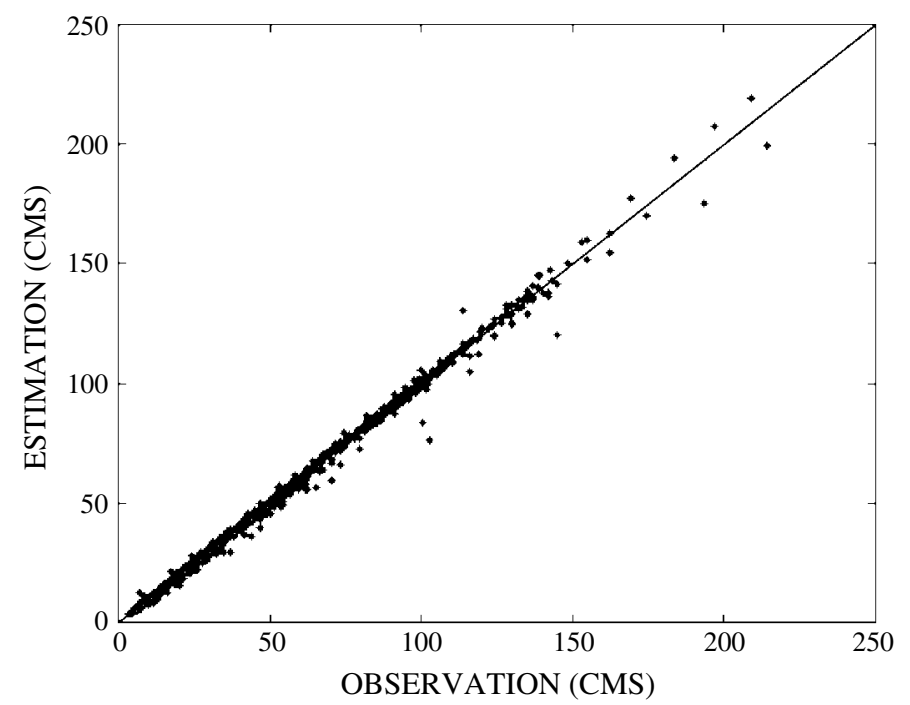

Figure 5. Comparison of observed and forecast stream flow at Son-Mou gauge in 1993

The processing layer of RTRL is constructed to have five nodes. Both learning rates ( $\eta_{1}$ and $\left.\eta_{2}\right)$ are $30 \cdot 0$. We found that after 30 steps on-line input training, RTRL can appropriately predict the one-hour ahead stream flow; that is, the network tends to be stable in its forecasting ability. The structures of ARMAX and RTRL are then applied to four different years without any further modifications. Summarized results of both methods are presented in Table VI. Apparently RTRL has a better performance than ARMAX. Figures 4 through to 8 show the observed and forecast streamflow by RTRL in 1992 to 1996, respectively. According to these 
results the network provides stable and precise predictions. The mean absolute error (MAE) is smaller than $0.8 \mathrm{cms}$ and the RMAE is less than $2.5 \%$. The result of one-step ahead prediction by RTRL in the case of typhoon Herb, which hit Taiwan in 1996 and was the largest typhoon in last 20 years, is presented in Figure 9. Although it has a slight time shift problem, the forecast, in general, is quite adequate.

\section{CONCLUSIONS}

The RTRL is an algorithm for training a completely recurrent, continually updated network to learn tasks. This feature is especially important for the extraordinary time variate characteristics of hydrological time-series. We

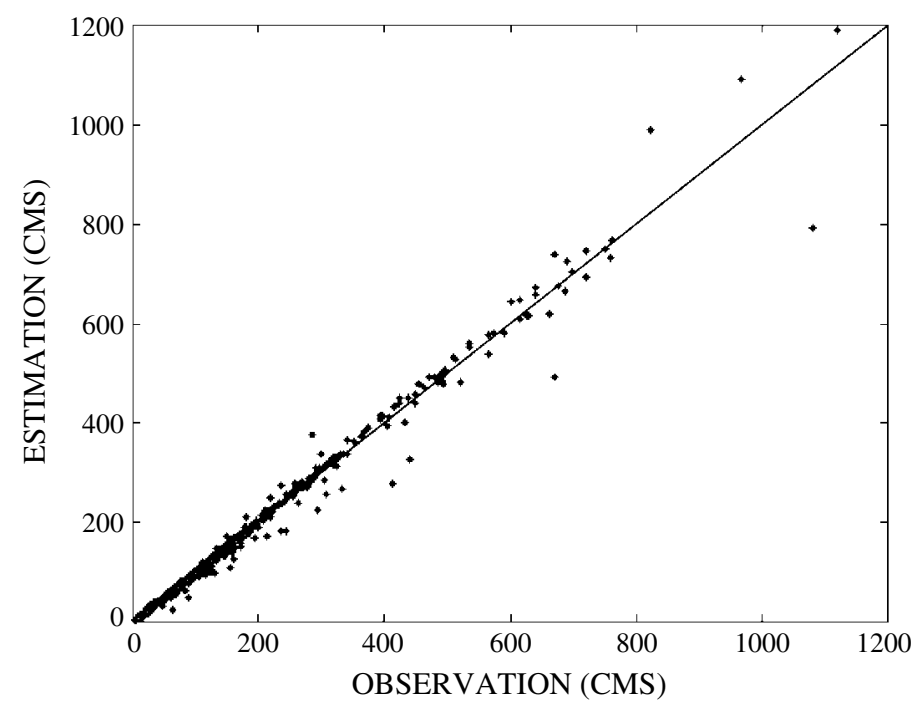

Figure 6. Comparison of observed and forecast stream flow at Son-Mou gauge in 1994

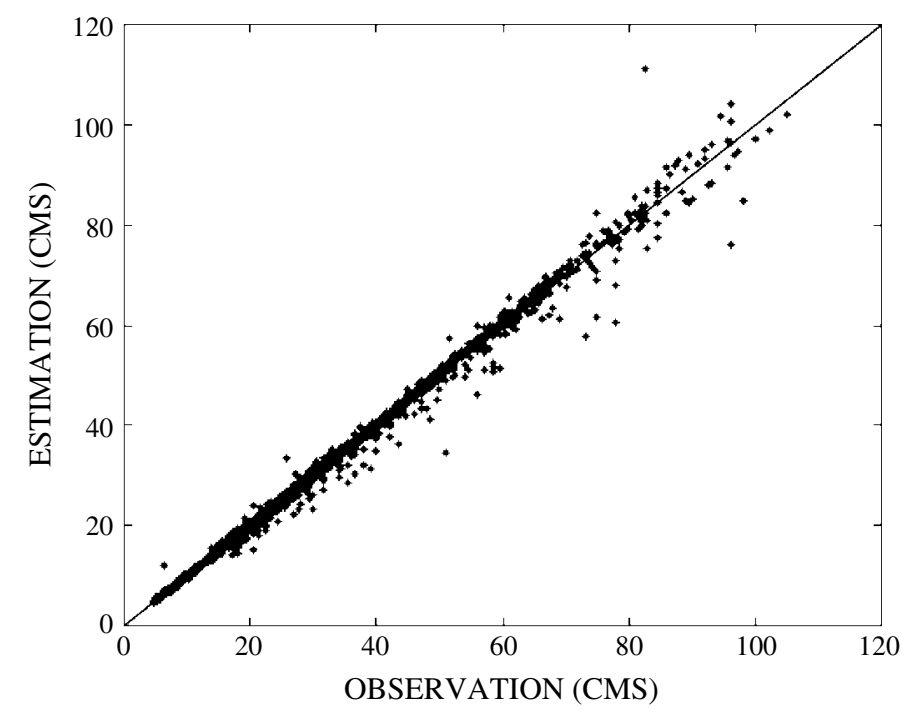

Figure 7. Comparison of observed and forecast stream flow at Son-Mou gauge in 1995 


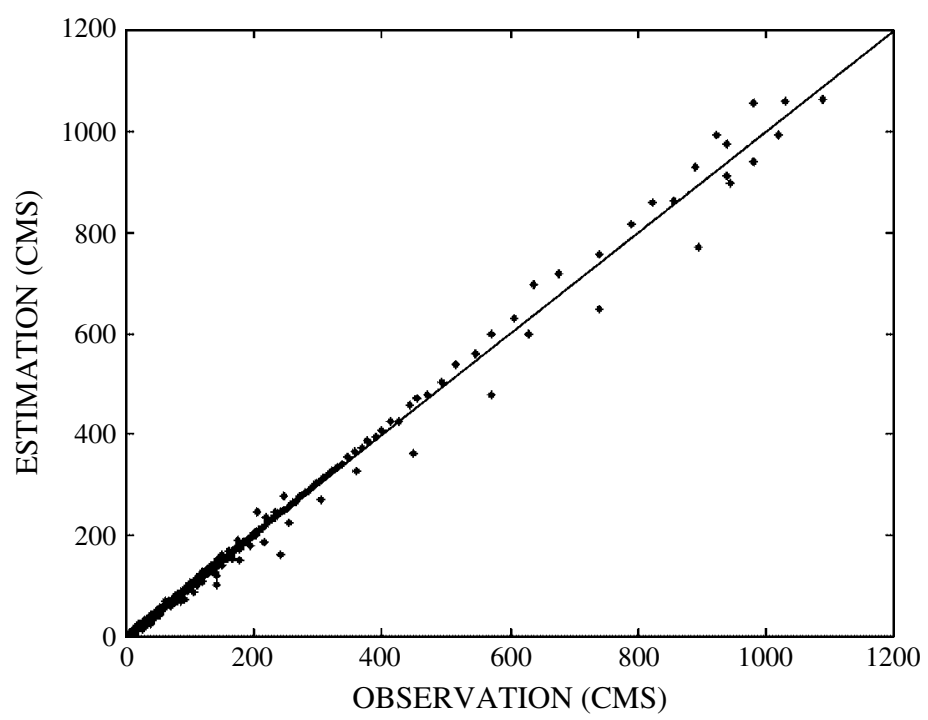

Figure 8. Comparison of observed and forecast stream flow in Son-Mou gauge in 1996

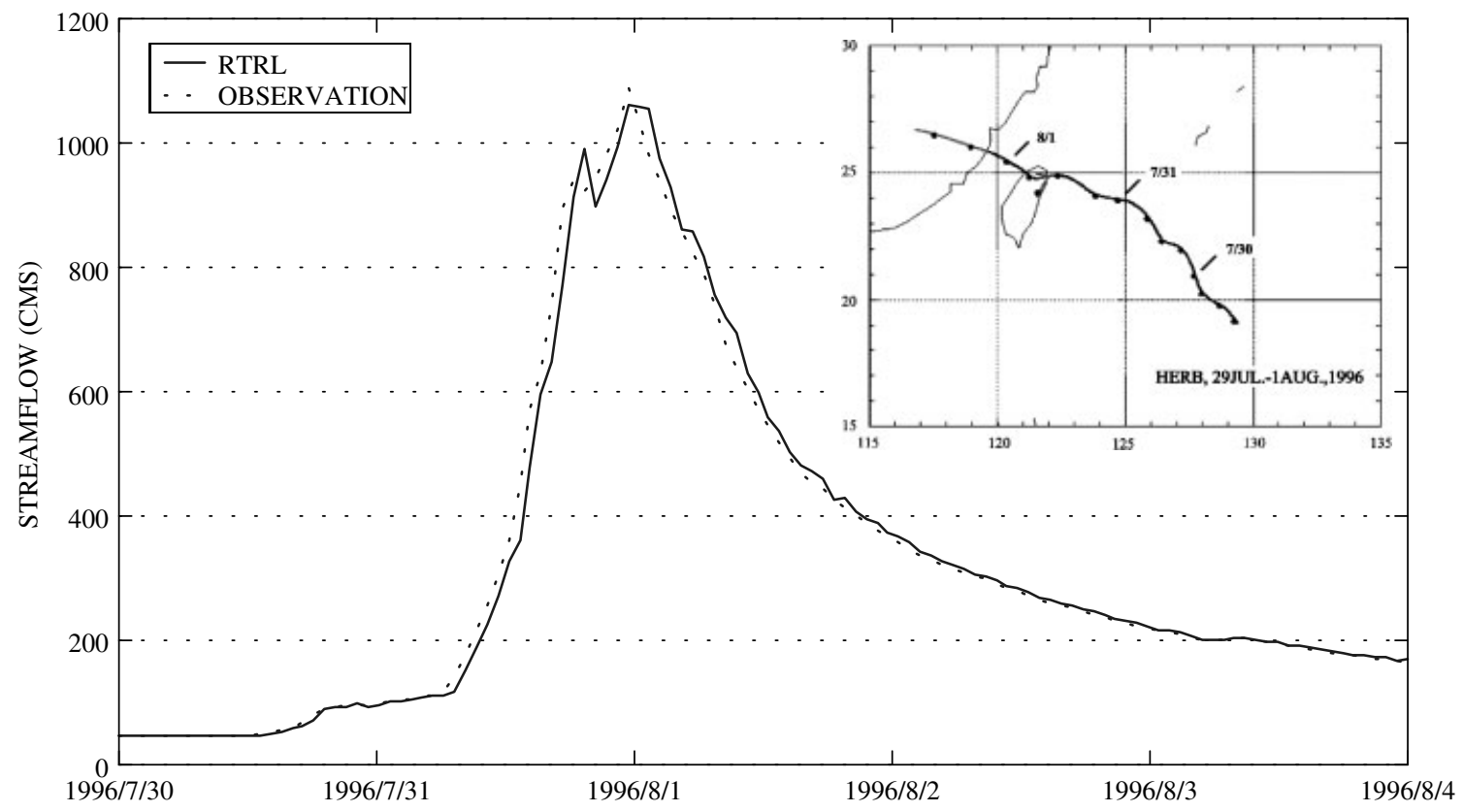

Figure 9. The observed and forecast stream flow at Son-Mou gauge during passage of Typhoon Herb in 1996

first use five different ARIMA time-series of simulation experiments to investigate the power and properties of this algorithm. The results indicate that the RTRL networks have similar performance to those of the fitted ARIMA models for one-step ahead prediction, and the networks could persist with the main statistical properties of the generated ARIMA series. This reveals the excellent learning ability and good performance of RTRL. Overall, RTRL is a well-suited model for time series that possess autoregressive moving average components. 
The rainfall and runoff data of Da-Chia river in Taiwan are used to demonstrate the practicability and applicability of RTRL for real-time stream-flow forecasting. A comparison of RTRL and ARMAX is also performed. The results show that (i) RTRL has better performance than ARMAX, and (ii) RTRL can be applied successfully to building a real-time stream-flow forecasting network with high accuracy. To deal with the one-step-ahead forecasting $\boldsymbol{x}(t+1)$, both on synthetic time-series and rainfall-runoff series, only current information $\boldsymbol{x}(t)$ is used as input. This provides evidence that the use of a dynamically driven feedback algorithm has the potential of reducing the memory requirement significantly.

\section{ACKNOWLEDGEMENT}

This paper is based on partial work supported by National Science Council, R.O.C. (Grant No. NSC89-2313B-002-041).

\section{REFERENCES}

Box GEP, Jenkins GM. 1976. Time Series Analysis Forecasting and Control, 2nd edn. Holden-Day: San Francisco.

Chang FJ, Chen YC. 2001. A counterpropagation fuzzy-neural network modeling approach to real-time stream-flow prediction. Journal of Hydrology 245: 153-164.

Chang FJ, Hwang YY. 1999. A Self-organization algorithm for real-time flood forecast. Hydrological Processes 13(2): 123-138.

Chang FJ, Hu HF, Chen YC. 2001. Counterpropagation fuzzy neural network for stream-flow reconstruction. Hydrological Processes 15(2): 219-232.

Haykin S. 1999. Neural Networks: a Comprehensive Foundation, 2nd edn. Prentice Hall: Upper Saddle River, NJ.

Hopfield JJ. 1984. Neuron with graded response have collective computational properties like those of two-state neurons. Proceedings of the National Academy of Sciences, USA 81: 3088-3092.

Hsu K-L, Gupta HV, Sorooshian S. 1995. Artificial neural network modeling of the rainfall-runoff process. Water Resources Research 31(10): 2517-2530.

Hydrological Engineering Center. 1990. HEC-1 Flood Hydrograph Package. Program Users Manual. U.S. Army Corps of Engineers: Davis, CA.

McCulloch WS, Pitts W. 1943. A logical calculus of the ideas immanent in nervous activity. Bulletin of Mathematical Biophysics 5: $115-133$.

Nie J, Linkens DA. 1994. Fast self-learning multivariable fuzzy controllers constructed from a modified CPN network. International Journal of Control 60(3): 369-393.

Rumelhart DE, Hinton GE, Williams RJ. 1986. Learning internal representation by error propagation. Parallel Distributed Processing 1: $318-362$.

Sajikumar N, Thandaveswara BS. 1999. A non-linear rainfall-runoff model using an artificial neural network. Journal of Hydrology 216: $32-55$.

Salas JD, Delleur JW, Yevjevich V, Lane WL. 1985. Applied Modeling of Hydrologic Time Series. Water Resources Publications: Littleton, Colorado.

Shamseldin AY. 1997. Application of a neural network technique to rainfall-runoff modelling. Journal of Hydrology 199: 272-294.

Sherman LK. 1932. Stream-Flow from rainfall by the unit-graph method. Engineering News Record 108: 501-505.

Williams R, Zipser D. 1989. A learning algorithm for continually running fully recurrent neural network. Neural Computation 1: $270-280$. 